\title{
(2) OPEN ACCESS \\ Clopidogrel can be an effective complementary prophylactic for drug-refractory migraine with patent foramen ovale
}

\author{
Yichen Guo ำ , Yujie Shi, Dan Zhu, Rui Liu, Yi Qi 두, Guogang Luo
}

Department of Neurology, The First Affiliated Hospital of Xi'an Jiaotong University, Xi'an, Shaanxi, China

\section{Correspondence to} Professor Guogang Luo, Department of Neurology, The First Affiliated Hospital of Xi'an Jiaotong University, Xi'an, Shaanxi 710061, China; Iguogang@163.com

Accepted 28 July 2020 Published Online First 26 August 2020

Check for updates

(c) American Federation for Medical Research 2020. Re-use permitted under CC BY-NC. No commercial re-use. Published by BMJ.

To cite: Guo Y, Shi Y,

Zhu D, et al. J Investig Med 2020;68:1250-1255.

\begin{abstract}
The present study aims to determine the potential prophylactic effect of clopidogrel for migraine with patent foramen ovale (PFO) in patients who are poor responders to two or more common preventive medications. Migraineurs underwent contrast-enhanced transcranial doppler examination to confirm the presence of PFO and determine the right-to-left shunt degree. Clopidogrel $75 \mathrm{mg} /$ day was added to the existing prophylactic regimen for 3 months and 6 months. The presence of PFO was found in $56.8 \%$ (151/266) of all patients with migraine and $70.2 \%$ (59/84) of migraine with aura $(\mathrm{MHA})$, and among MHA a large shunt was observed in 36 patients. Twenty-six patients with drugrefractory migraine took clopidogrel $75 \mathrm{mg} /$ day for 3 months. Compared with those at baseline, headache frequencies and attack durations were significantly lower $(6.17 \pm 3.93 /$ month $(M)$ vs $3.28 \pm 2.67 / M$, $p=0.003 ; 13.62 \pm 13.98 /$ hour $(H)$ vs $7.36 \pm 7.33 / H$, $p=0.0049$, respectively); visual analog scale scores and migraine disability assessment scores were also obviously decreased $(6.32 \pm 1.97$ vs $4.71 \pm 1.20$, $p<0.001 ; 22.14 \pm 7.13$ vs $16.00 \pm 5.92, p=0.001$, respectively). These improvements were maintained for 6 months in 12 patients. We concluded that PFO was closely correlated with migraine, especially in MHA. Clopidogrel could act as an effective complementary prophylactic for migraine with PFO in patients with poor response to routine prophylactics.
\end{abstract}

\section{INTRODUCTION}

Many studies have shown that the incidence of patent foramen ovale (PFO) is higher in patients with migraine than in those without migraine, ranging from $15 \%$ to $90 \%$, and is significantly higher in migraine with aura (MHA), with a prevalence of $16 \%$ to $90 \% .{ }^{12}$ Conversely, the prevalence of migraine with $\mathrm{PFO}$ is also higher than that in normal controls, ranging from approximately $16 \%$ to $64 \% .{ }^{2}$ Platelet aggregation and paradoxical embolization might link cardiac right-to-left shunts (RLS) to migraine. ${ }^{3}$

Many observational trials have suggested that migraine is alleviated after PFO closure, ${ }^{45}$ but the results of three prospective, randomized controlled trials-the MIST, PRIMA, and PREMIUM studies-did not report this result. $^{2} 67$ Some articles have shown that

\section{Significance of this study}

What is already known about this subject?

- The incidence of patent foramen ovale (PFO) is higher in patients with migraine than in those without migraine; migraine is alleviated after PFO closure; clopidogrel could improve new-onset migraine after device implantation.

What are the new findings?

- PFO was closely correlated with migraine, especially in migraine with aura(MHA); a large right-to-left shunt is very common in patients with MHA; clopidogrel could play an additional prophylactic role for migraine with PFO in patients with poor response to routine prophylactics.

\section{How might these results change the focus} of research or clinical practice?

- For the patients with PFO whom traditional drug therapy has not worked, compared with invasive surgical closure, we can firstly try to use clopidogrel to maximize improvement in patients with headache symptoms.

clopidogrel inhibits platelet aggregation and modulates the release of vasoactive substances, ${ }^{8}$ which could improve new-onset migraine after device implantation. ${ }^{9}$ Therefore, some scholars believe that clopidogrel administered early in the postoperative period plays a greater role in decreasing the number of migraine events than mechanical closure. ${ }^{10}$

We therefore conducted this study to evaluate the rate of PFO in patients with migraine and to determine the effect of clopidogrel on patients with drug-refractory migraine and PFO.

\section{PATIENTS AND METHODS}

This was a single-center non-randomized openlabel trial. From January 2015 to March 2017, the study was conducted in the headache clinic of the neurology department at the First Affiliated Hospital, Xi'an Jiao Tong University. The inclusion criteria for patients were as follows: (1) patients with migraine diagnosed according 
to the diagnostic criteria of both episodic migraine (EM) and chronic migraine (CM) in the 2013 International Classification of Headache Disorders third edition (ICHD, beta version $)^{11}$ and aged $18-65$ years, that CM defined as $\geq 15$ headache days/month averaged over the past 3 months and aged 18-65 years; (2) patients with no positive signs of neurological disorders and negative results on head CT or MRI; (3) patients with normal ECG, routine hematuria, and coagulation tests results; (4) patients with no history of medication overuse or drug allergy. The exclusion criteria were as follows: (1) patients with other types of primary headache; (2) patients with secondary headache; (3) patients with histories of hypertension, heart disease, diabetes, or other chronic diseases; (4) patients with somatization disorders or mental disorders; (5) patients with a history of chest or head trauma; (6) pregnant or lactating women. All of the subjects underwent contrast-enhanced transcranial doppler (cTCD) imaging to determine PFO and the size of the shunt. RLS was classified according to microbubble (MB) numbers: (1) $0 \mathrm{MBs}$ (negative result); (2) mild: 1-10 MBs; (3) moderate: $10<\mathrm{MBs} \leq 25$; (4) large: $>25$ MBs. $^{12}$

Then, we selected drug-refractory migraineurs from patients with PFO. Drug-refractory migraine was defined as $\geq 2$ headache attacks/month while on at least two classes of prophylactics, ${ }^{6}$ including $\beta$-blockers, anticonvulsants, calcium channel blockers, tricyclics, and serotonin antagonists, for at least 3 months. All of the patients were told of the possible preventive effects and potential side effects of clopidogrel, and they provided signed informed consent. Clopidogrel $75 \mathrm{mg}$ /day was added to the existing prophylactic migraine regimen for at least 3 months. Ibuprofen was used as an acute pain reliever for unbearable headache for not more than 2 days during the experimental period. All baseline information, including headache frequency, headache duration, Migraine Disability Assessment (MIDAS) score, and headache severity, was registered in detail. Headache severity was evaluated with the visual analog scale (VAS). After 3 months and 6 months, all of the enrolled patients were re-interviewed in the headache clinic; the above indicators were measured based on the patients' headache diaries.

The trial diagram is shown in figure 1 .

\section{STATISTICAL ANALYSIS}

Categorical variables are expressed as frequencies and percentages, and continuous variables are expressed as means \pm SDs. All data were tested for normality. The t-test and Wilcoxon's test were used to analyze differences between baseline data and that measured 3 or 6 months after clopidogrel treatment. One-way ANOVA or nonparametric tests were used to compare the differences among the data at baseline, 3 months and 6 months, and Bonferroni's multiple comparison test was used for post hoc tests. The p-value $<0.05$ was considered an acceptable statistically significant cut-off value.

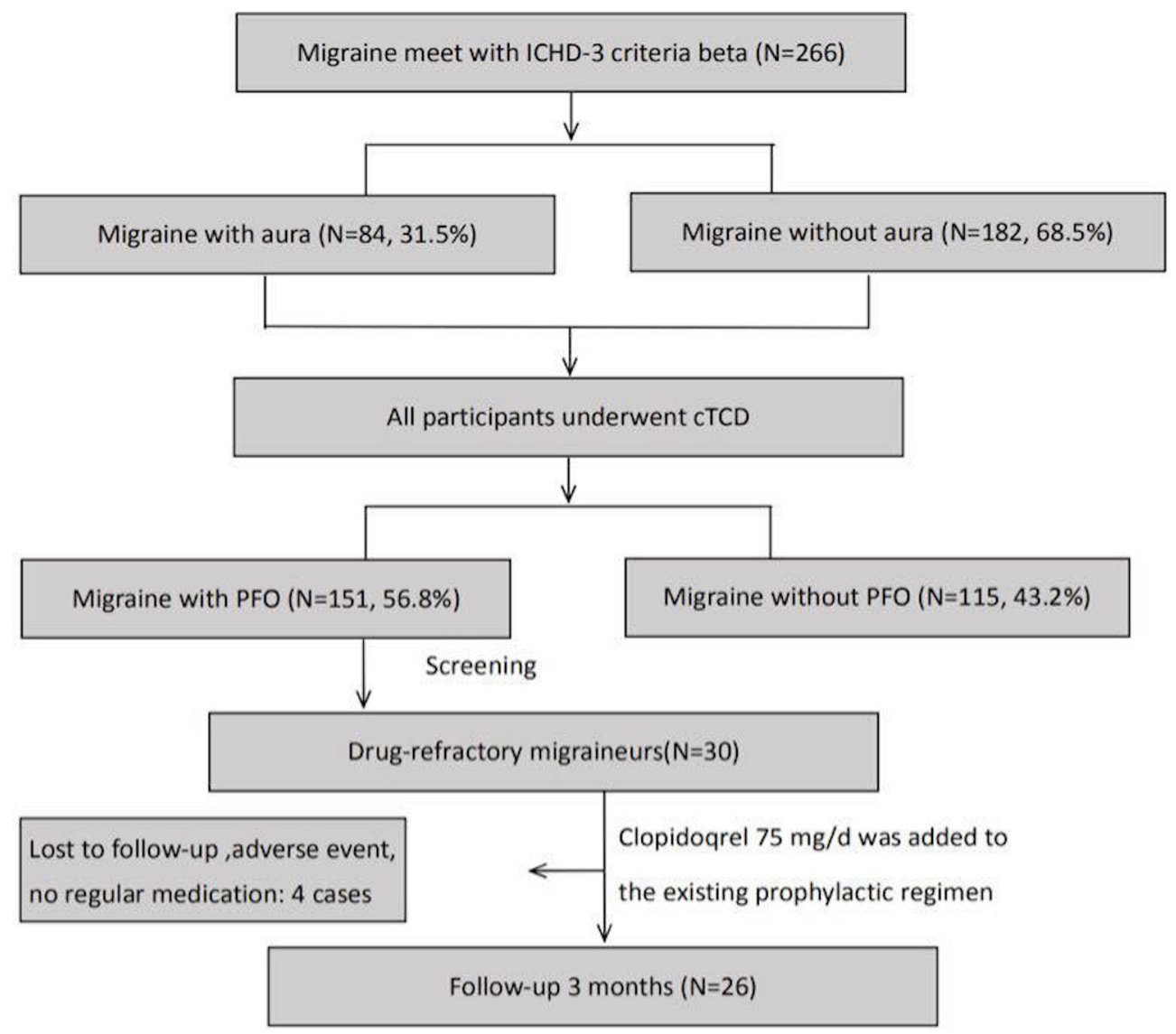

Figure 1 Trials diagram. CTCD, contrast-enhanced transcranial Doppler; ICHD-3, International Classification of Headache Disorders 3rd edition; PFO, patent foramen ovale. 
Table 1 Demographic characteristics of this study population

\begin{tabular}{llll}
\hline & $\begin{array}{l}\text { Migraine with } \\
\text { aura } \\
(\mathbf{n}=84)\end{array}$ & $\begin{array}{l}\text { Migraine without } \\
\text { aura } \\
(\mathbf{n}=182)\end{array}$ & $\begin{array}{l}\text { Migraine } \\
(\mathbf{n}=266)\end{array}$ \\
\hline $\begin{array}{l}\text { Sex F (\%) } \\
\text { Age }\end{array}$ & $36 / 84(66.7)$ & $113 / 182(62.1)$ & $169 / 266(63.5)$ \\
$\begin{array}{l}\text { Mean } \pm \text { SD (years) } \\
\text { Attack frequency (/ } \\
\text { month) }\end{array}$ & $3.37 \pm 1.24$ & $2.58 \pm 0.76$ & $3.02 \pm 1.14$ \\
$\begin{array}{l}\text { Headache duration } \\
\text { of each onset (/h) }\end{array}$ & $8.46 \pm 8.75$ & $6.38 \pm 10.39$ & $7.29 \pm 9.10$ \\
\hline $\begin{array}{l}\text { VAS scores } \\
\text { MIDAS scores }\end{array}$ & $4.28 \pm 1.47$ & $3.23 \pm 0.89$ & $3.70 \pm 1.26$ \\
\hline PFO+ (\%) & $10.56 \pm 3.78$ & $12.29 \pm 4.65$ & $10.93 \pm 2.49$ \\
\hline Large RLS & $36 / 84(70.2)$ & $92 / 182(50.5)$ & $151 / 266(56.8)$ \\
\hline
\end{tabular}

MIDAS, migraine disability assessment; PFO, patent foramen ovale; RLS, rightto-left shunt; VAS, visual analog scale.

\section{RESULTS}

The demographic characteristics of each group are presented in table 1. Visual aura was reported in 59 cases, sensory aura was reported in 18 cases, motor aura was reported in 5 cases, and speech/language symptoms was reported in 2 cases of MHA. The presence of PFO was observed in $56.8 \%(151 / 266)$ of all migraineurs and 65 patients with large shunts. Among these patients, the presence of PFO was observed in 70.2\% (59/84) of those with MHA, among whom a large shunt was observed in 36 patients. The presence of PFO was found in 50.5\% (92/182) of the migraine without aura (MoA) group, and a large shunt was observed in 29 patients.

Thirty drug-refractory migraineurs among the patients with PFO were screened for inclusion in the clopidogrel treatment study. Finally, 26 patients completed 3 months of treatment ( 3 patients were lost to follow-up, and 1 patient discontinued the intervention because of adverse events (she stopped taking clopidogrel due to increased menstruation)) (table 2). The headache frequencies and VAS scores conformed to normal distributions, and t-tests were used. Wilcoxon's tests were used to analyze headache attack durations and MIDAS scores since the data had nonnormal distributions. Compared with that at baseline, the headache frequency decreased from 6.17 $\pm 3.93 /$ month (M) to $3.28 \pm 2.67 / \mathrm{M}$, and the headache attack duration decreased from $13.62 \pm 13.98 /$ hour $(\mathrm{H})$ to $7.36 \pm 7.33 / \mathrm{H}$, with significant differences $(p=0.003 ; p=0.049)$. In addition, the VAS score and MIDAS score were also obviously lower than those at baseline $(6.32 \pm 1.97$ vs $4.71 \pm 1.20$, $\mathrm{p}<0.001 ; 22.14 \pm 7.13$ vs $16.00 \pm 5.92, \mathrm{p}=0.001$, respectively) (figure 2).

Twelve patients completed 6 months of follow-up. After testing for normality, the headache frequencies, VAS scores, and MIDAS scores were subjected to one-way ANOVA, and headache attack duration was analyzed by non-parametric tests. Bonferroni's multiple comparison test was used as the post hoc test. The headache frequency was lower at 3 $(3.62 \pm 6.38 / \mathrm{M})$ months or 6 months $(2.75 \pm 1.35 / \mathrm{M})$ than at baseline $(6.79 \pm 3.82 / \mathrm{M}, \mathrm{p}=0.002)$, with significant differences $(p=0.023 ; p=0.003)$. The headache attack duration was decreased after treatment $(17.00 \pm 14.05 / \mathrm{H}$
Table 2 Demographic characteristics of the patients with drugrefractory migraine with patent foramen ovale

\begin{tabular}{|c|c|c|c|}
\hline Patient & $\begin{array}{l}\text { Migraine } \\
\text { symptom }\end{array}$ & RLS & Prior medications used \\
\hline 1 & MHA & Large & Valproate, sumatripan \\
\hline 2 & MoA & Moderate & Flunarizine, gabapentin \\
\hline 3 & CM & Large & $\begin{array}{l}\text { Propranolol, amitriptyline, } \\
\text { naproxen }\end{array}$ \\
\hline 4 & MHA & Mild & Flunarizine, gabapentin \\
\hline 5 & MoA & Moderate & $\begin{array}{l}\text { Propranolol, amitriptyline, } \\
\text { topiramate, magnesium }\end{array}$ \\
\hline 6 & MoA & Mild & Sumatripan, propranolol \\
\hline 7 & MHA & Large & Flunarizine, lamotrigine \\
\hline 8 & MHA & Large & Flunarizine, topiramate \\
\hline 9 & MHA & Large & Metoprolol, valproate \\
\hline 10 & MoA & Mild & $\begin{array}{l}\text { Flunarizine, amitriptyline, } \\
\text { ibuprofen }\end{array}$ \\
\hline 11 & MoA & Moderate & Flunarizine, propranolol \\
\hline 12 & $\mathrm{CM}$ & Large & Divitamins, flunarizine, herb \\
\hline 13 & MoA & Mild & Propranolol, topiramate \\
\hline 14 & MoA & Mild & Topiramate, flunarizine \\
\hline 15 & MHA & Mild & Divitamins, amitriptyline \\
\hline 16 & MoA & Large & Flunarizine, valproate \\
\hline 17 & MoA & Moderate & Divitamins, topiramate \\
\hline 18 & MoA & Mild & Flunarizine, metoprolol \\
\hline 19 & MHA & Mild & Divitamins, amitriptyline \\
\hline 20 & MoA & Mild & Topiramate, aspirin, propranolol \\
\hline 21 & $\mathrm{CM}$ & Large & Flunarizine, paracetamol \\
\hline 22 & MoA & Large & Flunarizine, paracetamol \\
\hline 23 & MoA & Large & $\begin{array}{l}\text { Flunarizine, paracetamol, } \\
\text { ibuprofen }\end{array}$ \\
\hline 24 & MoA & Large & Flunarizine, paracetamol \\
\hline 25 & MHA & Large & Flunarizine, herb \\
\hline 26 & MHA & Large & Flunarizine, amitriptyline \\
\hline
\end{tabular}

$\mathrm{CM}$, chronic migraine; MHA, migraine with aura; MoA, migraine without aura; RLS, right-to-left shunt.

vs $7.41 \pm 6.74 / \mathrm{H}$ vs $6.95 \pm 5.36 / \mathrm{H}, \mathrm{p}=0.023)$, and did not show the statistical significance neither at 3 nor 6 months compared with baseline. The VAS scores $(6.50 \pm 2.26$ vs $4.70 \pm 0.94$ vs $4.45 \pm 1.07)$ and MIDAS scores $(27.08 \pm 5.61$ vs $20.33 \pm 5.46$ vs $16.67 \pm 4.46)$ were obviously reduced after clopidogrel treatment $(p=0.049 ; p=0.001$, respectively); the scores at 3 months $(p=0.0023 ; p=0.009$, respectively) and at 6 months $(p=0.008 ; p<0.001$, respectively) were lower than those at baseline. Interestingly, between 3 months and 6 months of treatment, there were no significant differences in the various headache indicators (figure 3).

\section{DISCUSSION}

cTCD imaging is a highly sensitive method for detecting the RLS. Its advantages are low cost and high sensitivity, which make it a common method for PFO screening in the clinic. $^{13}$

Some existing data have shown that PFO is a risk factor for migraine although research in this area has been quite scarce. This study used cTCD technology to examine the rate of $\mathrm{PFO}$ in more than 300 subjects to identify the 
A

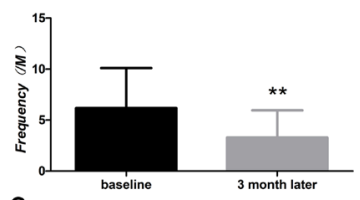

C

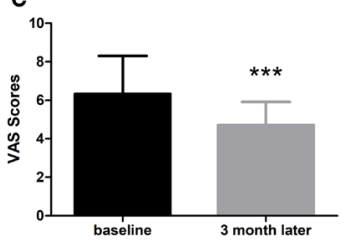

B

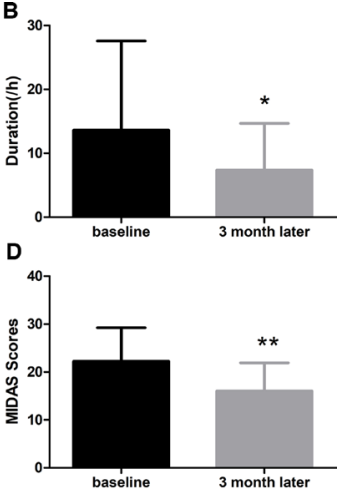

Figure 2 Comparison of frequency (A), duration (B), visual analog scale (VAS) score (C), and migraine disability assessment (MIDAS) score (D) between baseline and after clopidogrel treatment 3 months. Data are shown as means \pm SD. ${ }^{*} p<0.05$, ${ }^{* *} p<0.01,{ }^{* *} p<0.001$.

incidence of migraine associated with PFO and shunt size and the magnitudes of the relationships between migraine types so that readers interested in migraine associated with PFO could obtain a certain understanding.

We found PFO to be present in $56.8 \%$ of all of the patients with migraine. This result was similar to previously published findings ${ }^{14}$ in which the presence in patients with migraine was $25 \%$ higher than that in the general population. ${ }^{15}$ In our study, the prevalence rates of PFO were $70.2 \%$ in the MHA group and $50.5 \%$ in the MoA group, which were also similar to those in published studies. ${ }^{6}{ }^{16}$ In previously published reviews, Anzola et $a l^{17}$ and Choi et al ${ }^{18}$ reported that $\mathrm{PFO}$ was related to only $\mathrm{MHA}$. Tatlidede et $a l^{16}$ concluded that migraine, especially MHA, was closely related to PFO, as did Domitrz et al. ${ }^{19}$ In the current study, we estimated that the prevalence of PFO in migraineurs, especially those with MHA, was higher than those in the general population. The reason for this result has not yet

A

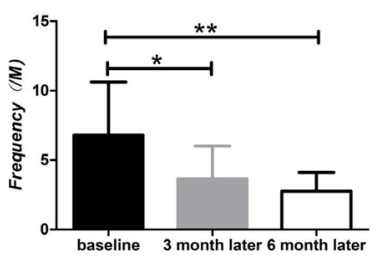

C

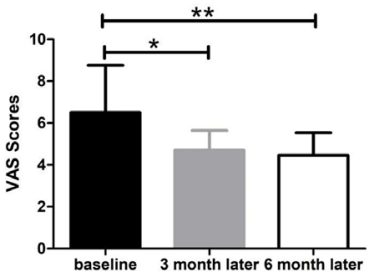

B

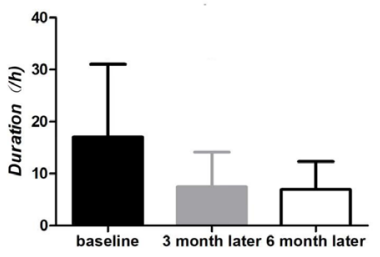

D

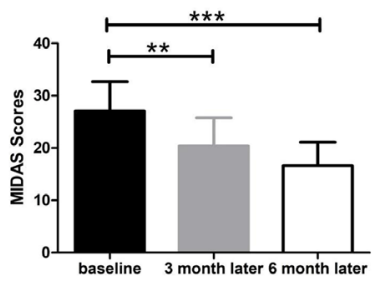

Figure 3 Comparison of frequency (A), duration (B), visual analog scale (VAS) score (C), and migraine disability assessment (MIDAS) score (D) between baseline and 3, 6 months later. Data are shown as means \pm SD. ${ }^{*} p<0.05,{ }^{* *} p<0.01,{ }^{* *} p<0.001$.

been clearly elucidated. It might be related to increased platelet activity and aggregation ability as well as abnormal embolism. ${ }^{320}$ Some researchers have proposed that migraine and atrial septal dysplasia might be attributable to similar autosomal inheritance. ${ }^{21}$ It has also been reported that the coexistence of PFO and migraine might due to a common occurrence during embryogenesis, but they are two lateral defects that are independent of each other. ${ }^{22}$

In patients with migraine with PFO, treatment with $\mathrm{PFO}$ occlusion has predominated in recent decades. Observational studies involving some small samples have found that transcatheter closure of PFO improves the frequency of migraine and the severity of headache. Confirming whether PFO closure is effective in the treatment of migraine requires large-sample randomized controlled trials (RCTs). Currently, three prospective RCTs, including the MIST, PRIMA, and PREMIUM studies, have published their results. None of these three studies reached its primary end point. In addition to surgical interventions, these studies considered antiplatelet drugs, in both the occlusion group and the drug/sham group experienced therapeutic effects, with no statistically significant difference. It could be concluded that occlusion was a beneficial supplement to medical treatment, but we boldly speculate that the alleviation of migraine in the RCTs may have been due to an antiplatelet effect.

Possible mechanisms of the relationship between migraine and PFO are the presence of RLS, increased platelet aggregation activity, microembolic signals, and 5 -hydroxytryptamine $(5-\mathrm{HT})$ in the venous circulation. 5 -HT is normally removed by the lungs; however, if it reaches the brain via circulation, it can induce local hypoxia and ischemia rather than stroke if it is present in sufficient concentrations. ${ }^{23}$ In response to the involvement of platelets in this process, we proposed prophylactic treatment of migraine with the antiplatelet drug clopidogrel bisulfate. Some scholars have suggested that clopidogrel should always be used routinely in the early postocclusion period. It is possible that the drug, rather than the occlusion device, could play a role in the relief of migraine. Wilmshuist et al's study found that the combination of clopidogrel for 1 month and aspirin for 6 months was superior to aspirin alone in preventing MHA after atrial occlusion. ${ }^{9}$ The mechanism of clopidogrel's long-term beneficial platelet effect, unlike that of aspirin, including platelet aggregation inhibition, suggesting that platelet activation might be involved in the pathogenesis of migraine, possibly by affecting the storage of 5-HT.

The characteristics of the enrolled patients were similar to those in the MIST ${ }^{6}$ and PRIMA $^{7}$ studies. All the patients had drug-refractory migraine and RLS. Due to the difficulty in enrolling patients with refractory migraine, it was challenging to enroll enough patients for matching, so our study was a self-controlled trial. In the overall migraine population, data including headache attack frequency, headache duration, and VAS and MIDAS scores should theoretically follow normal distributions. The non-normal distributions in this study may be due to the small sample size and poor overall representativeness. The headache durations and MIDAS scores of the sample were not normally distributed, so we used Wilcoxon's tests. Our study showed that headache symptoms were mitigated by the use of clopidogrel. 
At the end of the 3-month follow-up period, headache attack frequencies and durations and the VAS and MIDAS scores were significantly decreased compared with those at baseline. Some studies obtained similar positive conclusions. ${ }^{25-27}$ Only 12 patients completed all 6 months of treatment. Compared with the results at 3 months, the results at 6 months were not statistically significant. According to the results at the two time points, the improvement effect of clopidogrel seemed to reach its maximum effect at 3 months of treatment, and prolonging the medication course did not further improve headache symptoms. We think that this may be related to the small sample size. In addition, we speculate that with prolonged disease courses, patients experience anxiety and depression, and their threshold for pain may decrease, resulting in increased subjective feelings after the same intensity of pain stimulation.

Throughout therapeutic drug development history, clinical treatment guided by clear pathogenesis has been emphasized. Regarding the treatment of migraine, PFO has been a popular research topic. According to the results of the three published RCTs, surgical treatment for RLS in patients with refractory migraine seems to be of little significance. In addition, surgical closure is an invasive operation with risks of incision infection, arrhythmia, and cardiac tamponade, and the cost is relatively high. Our study found that clopidogrel hydrogen sulfate had some preventive effects in patients with a prolonged treatment course, but it did not further improve the symptoms of headache. Therefore, in patients for whom traditional drug therapy has not worked, clopidogrel hydrogen sulfate can be administered to maximize improvement in patients with headache symptoms.

There are obvious limitations in this study that must be further improved. First, due to the lack of a randomized control group, there may be certain selection bias, memory bias, and confounding, but the analysis of the benefits of the medication and its durations in multiple subjects rather than a single patient indicated a notable difference from the placebo effect. However, the sample size in this study was small, so generalization and the validity of the conclusions are limited.

\section{CONCLUSIONS}

PFO is closely correlated with migraine, especially MHA. A large RLS is very common in patients with MHA. Clopidogrel could play an additional prophylactic role in migraine with PFO. However, this result must be further verified in large-scale, case-controlled, double-blind, placebo-parallel clinical trials.

Acknowledgements The authors are grateful to Dr. Guoliang Li from the First Affiliated Hospital of Xi'an Jiaotong University for supporting our work. Thanks to all of the participating physicians and nurses.

Contributors YG: methodology, software, investigation, writing —original draft. YS: methodology, formal analysis, software. DZ: investigation, writing — review and editing. RL: resources, writing — review and editing. YQ: investigation, formal analysis. GL: conceptualization, resources, project administration, funding acquisition. All authors have read and agreed to the published version of the manuscript.

Funding This study was funded by the National Natural Science Foundation of China (No.81271227) and the National Key Technology R\&D Program of China (No. 2014BAI04B05).

Competing interests None declared.
Patient consent for publication Not required.

Ethics approval The study was approved by the Ethics Committee of the First Affiliated Hospital of Xi'an Jiaotong University (XJTU1AF-CRS-2016-019).

Provenance and peer review Not commissioned; externally peer reviewed.

Data availability statement The data used to support the findings of this study are available from the corresponding author on reasonable request.

Open access This is an open access article distributed in accordance with the Creative Commons Attribution Non Commercial (CC BY-NC 4.0) license, which permits others to distribute, remix, adapt, build upon this work noncommercially, and license their derivative works on different terms, provided the original work is properly cited, an indication of whether changes were made, and the use is non-commercial. See: http://creativecommons.org/ licenses/by-nc/4.0/.

\section{ORCID iDs}

Yichen Guo http://orcid.org/0000-0002-0417-9795

Yi Qi http://orcid.org/0000-0003-3687-735X

\section{REFERENCES}

1 Schwerzmann M, Nedeltchev K, Lagger F, et al. Prevalence and size of directly detected patent foramen ovale in migraine with aura. Neurology 2005;65:1415-8.

2 Tariq N, Tepper SJ, Kriegler JS, et al. Patent foramen ovale and migraine: closing the debate - a review. Headache 2016:56:462-78.

3 Nozari A, Dilekoz E, Sukhotinsky l, et al. Microemboli may link spreading depression, migraine aura, and patent foramen ovale. Ann Neurol 2010;67:221-9.

4 Reisman M, Christofferson RD, Jesurum J, et al. Migraine headache relief after transcatheter closure of patent foramen ovale. J Am Coll Cardiol 2005:45:493-5.

5 Schwerzmann M, Wiher S, Nedeltchev K, et al. Percutaneous closure of patent foramen ovale reduces the frequency of migraine attacks. Neurology 2004;62:1399-401.

6 Dowson A, Mullen MJ, Peatfield R, et al. Migraine intervention with STARFlex technology (MIST) trial: a prospective, multicenter, double-blind, shamcontrolled trial to evaluate the effectiveness of patent foramen ovale closure with STARFlex septal repair implant to resolve refractory migraine headache. Circulation 2008;117:1397-404.

7 Mattle HP, Evers S, Hildick-Smith D, et al. Percutaneous closure of patent foramen ovale in migraine with aura, a randomized controlled trial. Eur Heart J 2016;37:2029-36

8 Froldi G, Bertin R, Dorigo P, et al. Endothelium-independent vasorelaxation by ticlopidine and clopidogrel in rat caudal artery. J Pharm Pharmacol 2011;63:1056-62.

9 Wilmshurst PT, Nightingale S, Walsh KP, et al. Clopidogrel reduces migraine with aura after transcatheter closure of persistent foramen ovale and atrial septal defects. Heart 2005;91:1173-5.

10 Azarbal B, Tobis J, Suh W, et al. Association of interatrial shunts and migraine headaches: impact of transcatheter closure. J Am Coll Cardiol 2005;45:489-92.

11 Headache Classification Committee of the International Headache Society (IHS). The International classification of headache disorders, 3rd edition (beta version). Cephalalgia 2013;33:629-808.

12 Jauss M, Zanette E. Detection of right-to-left shunt with ultrasound contrast agent and transcranial Doppler sonography. Cerebrovasc Dis 2000;10:490-6.

13 Klötzsch C, Janssen G, Berlit P. Transesophageal echocardiography and contrast-TCD in the detection of a patent foramen ovale: experiences with 111 patients. Neurology 1994;44:1603-6.

14 Domitrz I, Mieszkowski J, Kamińska A. Relationship between migraine and patent foramen ovale: a study of 121 patients with migraine. Headache 2007:47:1311-8

15 Serafini 0 , Misuraca $G$, Siniscalchi A, et al. [Prevalence of aneurysm of the interatrial septum in the general population and in patients with a recent episode of cryptogenetic ischemic stroke: a tissue harmonic imaging transthoracic echocardiography study in 5.631 patients]. Monaldi Arch Chest Dis 2006;66:264-9.

16 Tatlidede AD, Oflazoğlu B, Celik SE, et al. Prevalence of patent foramen ovale in patients with migraine. Agri 2007;19:39-42.

17 Anzola GP, Magoni M, Guindani M, et al. Potential source of cerebral embolism in migraine with aura: a transcranial Doppler study. Neurology 1999:52:1622-5.

18 Choi DY, Shin DH, Cho KH, et al. Migraine with aura: a predictor of patent foramen ovale in children and adolescents. Cephalalgia 2013;33:463-8. 
19 Domitrz I, Styczynski G, Wilczko J, et al. An association between migraines and heart anomalies - true or false? A heart ultrasound study using CTTE in migraine patients and control participants. Pain Med 2014;15:2156-60.

20 Hershey AD, Tang Y, Powers SW, et al. Genomic abnormalities in patients with migraine and chronic migraine: preliminary blood gene expression suggests platelet abnormalities. Headache 2004;44:994-1004.

21 Kimmelstiel C, Gange C, Thaler D. Is patent foramen ovale closure effective in reducing migraine symptoms? A controlled study. Catheter Cardiovasc Interv 2007;69:740-6.

22 Kaaro J, Partonen T, Naik P, et al. Is migraine a lateralization defect? Neuroreport 2008;19:1351-3.
23 Post MC, Luermans JGLM, Plokker HWM, et al. Patent foramen ovale and migraine. Catheter Cardiovasc Interv 2007;69:9-14.

24 Denuelle M, Fabre N, Payoux P, et al. Posterior cerebral hypoperfusion in migraine without aura. Cephalalgia 2008;28:856-62.

25 Spencer BT, Qureshi Y, Sommer RJ. A retrospective review of clopidogrel as primary therapy for migraineurs with right to left shunt lesions. Cephalalgia 2014:34:933-7.

26 Sommer RJ, Nazif T, Privitera L, et al. Retrospective review of thienopyridine therapy in migraineurs with patent foramen ovale. Neurology 2018;91:1002-9.

27 Reisman AM, Robbins BT, Chou DE, et al. Ticagrelor for refractory migraine/ patent foramen ovale (TRACTOR): an open-label pilot study. Neurology 2018:91:1010-7. 